Article

\title{
Secure Resolving Sets in a Graph
}

\author{
Hemalathaa Subramanian ${ }^{1, *}$ and Subramanian Arasappan ${ }^{2}$ \\ 1 Research Scholar, Register No. 10445, The M.D.T. Hindu College, Tirunelveli 627010 Affiliated to \\ Manonmaniam Sundaranar University, Abishekapatti, Tirunelveli 627 012, Tamilnadu, India \\ 2 Principal, Department of Mathematics, The M.D.T. Hindu College, Tirunelveli 627 010, India; \\ asmani1963@gmail.com \\ * Correspondence: hemarmath@gmail.com
}

Received: 5 September 2018; Accepted: 19 September 2018; Published: 27 September 2018

check for updates

\begin{abstract}
Let $G=(V, E)$ be a simple, finite, and connected graph. A subset $S=\left\{u_{1}, u_{2}, \ldots, u_{\mathrm{k}}\right\}$ of $V(G)$ is called a resolving set (locating set) if for any $x \in V(G)$, the code of $x$ with respect to $S$ that is denoted by $C_{S}(x)$, which is defined as $C_{S}(x)=\left(d\left(u_{1}, x\right), d\left(u_{2}, x\right), . ., d\left(u_{k}, x\right)\right)$, is different for different $x$. The minimum cardinality of a resolving set is called the dimension of $G$ and is denoted by $\operatorname{dim}(G)$. A security concept was introduced in domination. A subset $D$ of $V(G)$ is called a dominating set of $G$ if for any $v$ in $V-D$, there exists $u$ in $D$ such that $u$ and $v$ are adjacent. A dominating set $D$ is secure if for any $u$ in $V-D$, there exists $v$ in $D$ such that $(D-\{v\}) \cup\{u\}$ is a dominating set. A resolving set $R$ is secure if for any $s \in V-R$, there exists $r \in R$ such that $(R-\{r\}) \cup\{s\}$ is a resolving set. The secure resolving domination number is defined, and its value is found for several classes of graphs. The characterization of graphs with specific secure resolving domination number is also done.
\end{abstract}

Keywords: resolving set; domination; secure resolving set and secure resolving domination

\section{Introduction}

Let $G=(V, E)$ be a simple, finite, and connected graph. Let $S=\left\{u_{1}, u_{2}, \ldots, u_{\mathrm{k}}\right\}$ on which the ordering $\left(u_{1}, u_{2}, \ldots, u_{\mathrm{k}}\right)$ is imposed. For any $w \in V(G)$, the ordered $k$-tuples $r(w \mid S)=\left(d\left(u_{1}, w\right)\right.$, $\left.d\left(u_{2}, w\right), \ldots, d\left(u_{\mathrm{k}}, w\right)\right)$ is known as the metric description of $w$ with respect to $S$. The set $S$ is called a resolving set of $G$ if $r(u \mid S)=r(w \mid S)$ implies $u=w$ for all $u, w \in V(G)$. A resolving set of $G$ of minimum cardinality is called a minimum resolving set or a basis, and the cardinality of a minimum resolving set is called the dimension of $G$, which is denoted by $\operatorname{dim}(G)$ [1].

The idea of locating sets in a connected graph is already available in the literature [2,3]. Slater initiated the concept of locating sets (resolving sets) and a reference set (metric dimension) nearly four decades ago. Later, Harary and Melter found the above-mentioned theory [4] independently. They adopted the term metric dimension for locating number. Several papers have been published on resolving sets, resolving dominating sets, independent resolving sets, etc.

Security is a concept that is associated with several types of sets in a graph. For example, a dominating set $D$ of $G$ is secure set if for any $v \in V-D$ there exist $u \in D$ such that $(D-\{u\}) \cup\{v\}$ is a dominating set $[5,6]$. Secure independent sets, secure equitable sets etc., have been defined and discussed. In this paper, secure resolving sets and secure resolving dominating sets are introduced and studied.

In this paper, $G$ refers to a simple, finite, and connected graph. The abbreviations used in this paper are as follows:

- $\quad$ SR set: Secure resolving set

- $\quad$ SRD set: Secure resolving dominating set 
The role of symmetry in the following study:

Regarding the symmetry role, a complete graph has vertex transitivity, which is a symmetry. $K_{m, n}$ has vertices that have degree symmetry in the partite sets. One more important aspect of symmetry is present in the concept of the SR set, as well as in SRD sets. In SR sets, every vertex has the opportunity of being a member of a resolving set. Thus, a symmetry is achieved in the presence of vertices. A similar thing happens in domination. In practical application, in any Executive Council, equal opportunity is to be given to all of the members of the General Council for inclusion in the Executive Council. Thus, the spirit of symmetry is present in the form of equality. That is, there is a symmetry in the treatment of vertices.

\section{Secure Resolving Dimension}

Definition 1. A subset $T$ of $G$ is a SR set of $G$ if $T$ is resolving and for any $x \in V-T$, there exists $y \in T$ such that $(T-\{y\}) \cup\{x\}$ is a resolving set of $G$. The minimum cardinality of a SR set of $G$ is known as the secure resolving dimension of $G$, and is marked by $\operatorname{sdim}(G)$.

Remark 1. The existence of a SR set is guaranteed.For, in any graph, the vertex set $V(G)$ is a secure set as well as a resolving set.

Remark 2. $\operatorname{dim}(G) \leq \operatorname{sdim}(G)$.

\section{Secure Resolving Dimension for Some Well-Known Graphs}

1. $\operatorname{sdim}\left(K_{n}\right)=n-1=\operatorname{dim}\left(K_{n}\right)$

2. $\operatorname{sdim}\left(K_{1, n}\right)=n>\operatorname{dim}\left(K_{1, n}\right)$

3. $\operatorname{sdim}\left(K_{m, n}\right)=m+n-2=\operatorname{dim}\left(K_{m, n}\right)(m, n \geq 2)$

4. $\operatorname{sdim}\left(P_{n}\right)=2>\operatorname{dim}\left(P_{n}\right)=1(n \geq 3)$

5. $\operatorname{sdim}\left(C_{n}\right)=2=\operatorname{dim}\left(C_{n}\right)$

6. $\operatorname{sdim}\left(K_{m}\left(a_{1}, a_{2}, \ldots, a_{m}\right)\right)=\left\{\begin{array}{c}\operatorname{dim}\left(K_{m}\left(a_{1}, a_{2}, \ldots, a_{m}\right)\right)+1 \\ \text { if } a_{i} \geq 2 \text { for atleast one } i \\ \operatorname{dim}\left(K_{m}\left(a_{1}, a_{2}, \ldots, a_{m}\right)\right) \\ \text { if } a_{i}=1 \text { for all } i .\end{array}\right.$

where $\left(K_{m}\left(a_{1}, a_{2}, \ldots, a_{\mathrm{m}}\right)\right)$ is the multi-star graph formed by joining $a_{i} \geq 1(1 \leq i \leq m)$ pendant vertices to each vertex $x_{i}$ of a complete graph $K_{m}$ with $V\left(K_{m}\right)=\left\{x_{1}, x_{2}, \ldots, x_{m}\right\}$.

Illustration 1. Consider $C_{5}$.

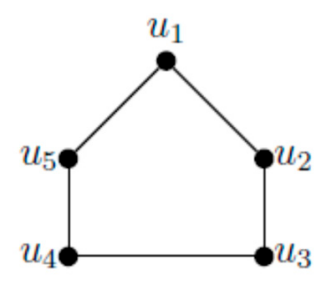

let $H=\left\{u_{1}, u_{3}\right\}$. Then, $H$ is resolving, and for any $u \in V-H$, there exists $v \in H$ such that $(H-\{v\}) \cup$ $\{u\})$ is a resolving set of $C_{5}$. It can be easily seen that $\operatorname{sdim}(G)=2$.

\section{Secure Resolving Dimension for Special Classes of Graphs}

Observation 1. Let order of $G \geq 3$. Suppose $\operatorname{sdim}(G)=1$. Then, $\operatorname{dim}(G)=(\operatorname{since} \operatorname{sdim}(G) \geq \operatorname{dim}(G))$. Therefore, $G=P_{n}$. However, $\operatorname{sdim}\left(P_{n}\right)=2$, which is a contradiction. Therefore, $\operatorname{sdim}(G) \geq 2$.

Observation 2. $\operatorname{sdim}(G)=1$ if and only if $G=P_{1}$ or $P_{2}$. 
Theorem 1. $\operatorname{sdim}(G)=2, G$ is a tree if and only if $G=P_{n}(n \geq 3)$.

Proof. If $G=P_{n}(n \geq 3)$, then $\operatorname{sdim}(G)=2$.

Conversely, suppose $n \geq 4$. Suppose there are two pendant vertices $v_{1}, v_{2}$ adjacent with $w$ of $G$. Take a vertex $t$, which is adjacent to $w\left(t \neq v_{1}, v_{2}\right) .\left\{v_{1}, w\right\}$ is not a resolving set, since $t$ and $v_{2}$ will not have distinct codes with respect to $\left\{v_{1}, w\right\}$. Assume that $\left\{v_{1}, v_{2}\right\}$ is a resolving set of $\mathrm{G}$. Then, it is not secure, since $\left\{v_{1}, w\right\}$ and $\left\{v_{2}, w\right\}$ are not resolving sets. Suppose $\left\{v_{1}, t\right\}$ is resolving. Then, it is not secure (since $\left\{v_{1}, w\right\},\{w, t\}$ are not resolving sets of $\left.G\right)$. Let $\{a, b\}$ be a resolving set of $G . a, b \notin\left\{v_{1}, v_{2}, w\right\}$. Then, $\{a, b\}$ is not secure, since neither $\{w, b\}$ nor $\{w, a\}$ is a resolving set of $P_{n}$ (since $d\left(v_{1}, a\right)=d(w, a)+1=d\left(v_{2}, a\right)$ ). Therefore, no vertex of $G$ supports two or more pendant vertices. Suppose that $w$ is a vertex of $G$ that supports one pendant vertex and there exists at least two neighbors of $w$ having degrees greater than or equal to two. Then, we will not get any resolving set with cardinality two containing $w$. Therefore, any vertex of $G$ with a pendant neighbor has at most one neighbor of degree greater than or equal to two. Therefore, $G$ is a path. Suppose that $n=3$. Since $G$ is acyclic and connected, $G=P_{3}$.

Theorem 2. $\operatorname{sim}\left(C_{n}\right)=\operatorname{dim}\left(C_{n}\right)=2$.

Proof. Let $V\left(C_{n}\right)=\left\{m_{1}, m_{2}, \ldots, m_{n}\right\}$.

Case (i): $n=2 k+1$.

Let $M=\left\{m_{1}, m_{2}\right\}$. Then, $M$ is a resolving set of $C_{n}$. It can be verified that $\left\{m_{1}, m_{i}\right\}$ is a resolving set where $3 \leq i \leq n$.

Case (ii): $n=2 k$.

Then, $m_{1}$ and $m_{k}$ are diametrically opposite vertices. Let $M=\left\{m_{1}, m_{2}\right\}$. Clearly, $\left\{m_{1}, m_{2}\right\}$ is a resolving set of $C_{n}$. It can be substantiated that $\left\{m_{1}, m_{i}\right\}$ is resolving when $3 \leq i \leq n, i \neq k$. Also, $\left\{m_{k}\right.$, $\left.m_{2}\right\}$ is a resolving set of $C_{n}$. Therefore, $\operatorname{sdim}(G)=2=\operatorname{dim}(G)$.

Remark 3. $\operatorname{sdim}(G) \leq 1+\operatorname{dim}(G)$

Proof. $\operatorname{sdim}(G) \geq \operatorname{dim}(G)$. Suppose that $\operatorname{dim}(G)<\operatorname{sdim}(G)$. Let $T=\left\{u_{1}, u_{2}, \ldots, u_{k}\right\}$ be a basis of $G$. Let $W=$ $\left\{u_{1}, u_{2}, \ldots, u_{k}, v\right\}$. Then, $W$ is a SR set of $G$. Therefore, $\operatorname{sdim}(G) \leq k+1$. However, $\operatorname{sdim}(G)>\operatorname{dim}(G)=k$. Therefore, $\operatorname{sdim}(G) \leq 1+\operatorname{dim}(G)$. Hence the remark.

Theorem 3. $\operatorname{sdim}(G)=n-1$ if and only if $G=K_{n}$ or $K_{1, n-1}$.

Proof. Let $G=K_{n}$ or $K_{1, n-1}$. Then, $\operatorname{sdim}(G)=n-1$. Suppose that $\operatorname{sdim}(G)=n-1$. Then, $\operatorname{dim}(G)$ is $n-1$ or $n-2$. If $\operatorname{dim}(G)=n-1$, then $G=K_{n}$. Suppose that $\operatorname{dim}(G)$ is $n-2$. Then, $G=K_{a, b}(a, b \geq 1), \mathrm{Ka}+\overline{\mathrm{Kb}}$ $(b \geq 2, a \geq 1), K_{a}+\left(K_{1} \cup K_{b}\right)(b, a \geq 1)$. Suppose that $G=K_{a, b}(a, b \geq 2), \operatorname{sdim}(G)=\operatorname{dim}(G)=a+b-2[1]$. Suppose that $G=\mathrm{Ka}+\overline{\mathrm{Kb}}(b \geq 2, a \geq 1)$, then $\operatorname{dim}(G)=a+b-2$. If $a=1$, then $\mathrm{Ka}+\overline{\mathrm{Kb}}=K_{1, b}$. In this case, $\operatorname{sim}(G)=b$ and $\operatorname{dim}(G)=b-1$. If $a \geq 2$, then $\operatorname{sim}(G)=a+b-2=\operatorname{dim}(G)$.

Let $G=K_{a}+\left(K_{1} \cup K_{b}\right),(a, b \geq 1)$. When $a=1$ and $b=1, G=P_{3}$ and $\operatorname{sim}\left(P_{3}\right)=2$ and $\operatorname{dim}\left(P_{3}\right)=1$. Clearly, $G$ is a star. When $a=1$ and $b \geq 2, \operatorname{sim}(G)=a+b-1=\operatorname{dim}(G)$. Suppose that $a>1$ and $b=1$. Then, $\operatorname{sim}(G)=a=\operatorname{dim}(G)$. Suppose that $a, b>1$. Then, $\operatorname{sim}(G)=a+b-1=\operatorname{dim}(G)$. Except when $G$ is a star, $\operatorname{sim}(G)=\operatorname{dim}(G)=n-2$. Therefore, $G=K_{1, n-1}$.

Theorem 3. Let $T$ be a connected graph. Let $G=T K_{2}$. Then, $\operatorname{sdim}(T) \leq \operatorname{sdim}\left(T K_{2}\right) \leq \operatorname{sdim}(T)+1$.

Proof. Refer to Theorem 7 [1]. Let $G=T K_{2}, T_{1}$, and $T_{2}$ be the transcripts of $T$ in $G$. Let $X$ be a basis of $T$, and let $X_{1}=\left\{x_{1}, x_{2}, \ldots, x_{k}\right\}$ and $X_{2}=\left\{y_{1}, y_{2}, \ldots, y_{k}\right\}$ be the basis of $T_{1}$ and $T_{2}$ respectively, corresponding to $X$. Let $S=X_{1} \cup\left\{y_{1}\right\}$. Then, $S$ is a SR set of $G$. Therefore, $\operatorname{sdim}(G) \leq \operatorname{sdim}(T)+1$. Let $V_{1}$, 
$\mathrm{V}_{2}$ be the vertex sets of $T_{1}$ and $T_{2}$ respectively. Then, $V(G)=V_{1} \cup V_{2}$. Let $X$ be a secure basis of $G$. Let $X_{1}=X \cap V_{1}, X_{2}=X \cap V_{2}$. Let $S_{1} \subseteq V\left(T_{1}\right)$ be the union of $X_{1}$ and the set $X_{2}^{\prime}$ consisting of those vertices of $V_{1}$ corresponding to $X_{2}$. Then, $S_{1}$ is a SR set of $T_{1}$. Therefore, $\operatorname{sdim}(T)=\operatorname{sdim}\left(T_{1}\right) \leq\left|S_{1}\right|=$ $\left|X_{1} \cup X_{2}^{\prime}\right| \leq\left|X_{1}\right|+\left|X_{2}^{\prime}\right|=|X|=\operatorname{sdim}(G)$. Hence, the theorem.

Corollary 1. Let $\epsilon>0$. Then, $\frac{\operatorname{sim}(G)}{\operatorname{sdim}(T)}<\epsilon$ where $T$ is a connected induced subgraph of $G$.

Proof. Let $T=K_{1}, 2^{n+1}$. $\operatorname{sdim}(T)=2^{n+1}$. Let $G=T K_{2}$. Then, we get a graph $G$ containing $T$ as an induced subgraph [1]. Further, $\operatorname{sdim}(G) \leq 2 n$. Therefore, $\frac{\operatorname{sim}(G)}{\operatorname{sdim}(T)} \leq \frac{2 n}{2^{n+1}} \rightarrow 0$ as $n \rightarrow 0$. Hence, the corollary.

\section{Secure Resolving Domination Number}

Definition 2. Let $U$ be a subset of $G$. $U=\left\{u_{1}, u_{2}, \ldots, u_{k}\right\}$ of $V(G)$ is said to be a SRD set of $G$ if $U$ is a dominating set of $G, U$ is resolving, and $U$ is secure. The minimum cardinality of a SRD set of $G$ is known as a secure resolving domination number of $G$, and is represented by $\gamma_{s r}(G)$.

Remark 4. $V$ is a $S R D$ set of $G$.

\section{Secure Resolving Domination Number for Some Well-known Graphs}

1. $\gamma_{s r}\left(K_{n}\right)=n-1, n \geq 2$.

2. $\gamma_{s r}\left(K_{1, n-1}\right)=n-1, n \geq 2$.

3. $\gamma_{s r}\left(P_{n}\right)=\left\{\begin{array}{cc}2 & \text { if } n=3,4 \\ \left\lceil\frac{n}{3}\right\rceil+1 & \text { if } n \geq 5 .\end{array}\right.$

4. $\quad \gamma_{s r}\left(C_{\mathrm{n}}\right)=\left\{\begin{array}{cc}2 & \text { if } n=3,4 \\ \left\lceil\frac{n}{3}\right\rceil+1 & \text { if } n \geq 5 .\end{array}\right.$

5. $\gamma_{s r}\left(K_{a_{1}, a_{2}, \ldots, a_{m}}\right)=\left(a_{1}+a_{2}+\ldots+a_{m}\right)-m$.

6. $\gamma_{s r}\left(K_{m}\left(a_{1}, a_{2}, \ldots, a_{m}\right)\right)=\left\{\begin{array}{c}m+a_{k+1}+\ldots+a_{m}-k \\ \text { if } a_{1}=\ldots=a_{k}=1 a_{i} \geq 2, k+1 \leq i \leq m \\ m \text { if } a i=1 \text { for all } i\end{array}\right.$

where $\left(K_{m}\left(a_{1}, a_{2}, \ldots, a_{m}\right)\right)$ is the multi-star graph formed by joining $a_{i} \geq 1(1 \leq i \leq m)$ pendant vertices to each vertex $x_{i}$ of a complete graph $K_{m}$ with $V\left(K_{m}\right)=\left\{x_{1}, x_{2}, \ldots, x_{m}\right\}$.

Illustration 2. Consider the following graph $K_{3}(1,1,1)$.

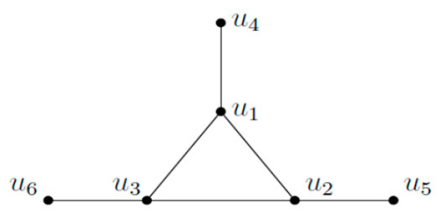

let $N=\left\{u_{1}, u_{2}, u_{3}\right\}$. Then $N$ is a secure, dominating, and resolving set of $K_{3}(1,1,1)$. It can be easily seen that. $\gamma_{s r}\left(K_{3}(1,1,1)\right)=3$.

Proposition 1. Let $\gamma_{s}$ be the minimum cardinality of a secure dominating set of $G$. Then, $\max \left\{\gamma_{s}(G), \operatorname{dim}(G)\right.$, $\left.\gamma_{r}(G)\right\} \leq \gamma_{s r}(G) \leq \gamma_{s}(G)+\operatorname{dim}(G)$.

Proof. Let $L$ be a minimum secure dominating set of $G$ and $W$ be a basis of $G$. Then, $L \cup W$ is a SRD set of $G$. Hence, $\gamma_{s r}(G) \leq \gamma_{s}(G)+\operatorname{dim}(G)$. The first inequality is obvious.

Remark 5. $P \cup\{u\}$ is a $S R D$ set of $G, P$ is a minimum resolving dominating set of $G$. 
Illustration 3. Consider the given graph $G$.

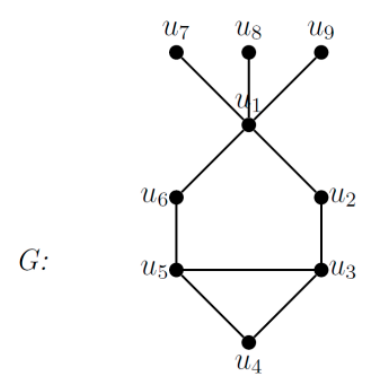

Here, $\gamma(G)=2$ and $\operatorname{dim}(G)=3$ (since $\left\{u_{1}, u_{4}\right\}$ is a minimum dominating set, $\left\{u_{5}, u_{7}, u_{8}\right\}$ is a minimum resolving set of $G$ ). $\gamma_{r}(G)=4$ (since $\left\{u_{1}, u_{5}, u_{7}, u_{8}\right\}$ is a minimum resolving dominating set of $G$. $\left\{u_{1}\right.$, $\left.u_{4}, u_{5}, u_{7}, u_{8}\right\}$ is a SRD set of $G$. Let $S$ be a minimum SRD set of $G$. Consequently, $\gamma_{s r}(G) \leq 5$. Since $S$ is resolving, $S$ must contain two of the pendant vertices. If $S$ contains $u_{2}$, then $u_{6}$ and the remaining pendant vertices are not resolved. If $S$ contains $u_{6}$, then $u_{2}$ and the remaining pendant vertices are not resolved. If $S$ contains $u_{4}$, then $u_{5}$ and $u_{3}$ are not resolved. Therefore, either $S$ contains $u_{5}$ and two of the pendant vertices or $u_{3}$ and two of the pendant vertices. If $S$ contains $u_{5}$ and two of the pendant vertices, then the remaining pendant vertex is not resolved. Therefore, the resolving dominating set contains $u_{1}$. Therefore, the possibilities of the resolving dominating sets are $\left\{u_{1}, u_{5}, u_{7}, u_{8}\right\},\left\{u_{1}, u_{5}, u_{8}\right.$, $\left.u_{9}\right\},\left\{u_{1}, u_{5}, u_{7}, u_{9}\right\},\left\{u_{1}, u_{3}, u_{7}, u_{8}\right\},\left\{u_{1}, u_{3}, u_{8}, u_{9}\right\}$, and $\left\{u_{1}, u_{3}, u_{7}, u_{9}\right\}$. None of these is secure, since $u_{2}$ and $u_{6}$ cannot be replaced in all of these sets. Therefore, $\gamma_{s r}(G) \geq 5$. Hence, $\gamma_{s r}(G)=5$. Thus, $\gamma(G)<$ $\operatorname{dim}(G)<\gamma_{r}(G)<\gamma_{s r}(G)$. Also, $\gamma_{s r}(G)=\gamma(G)+\operatorname{dim}(G) \cdot \gamma_{s}(G)=3$, since $G$ has no secure dominating set with two vertices, and $\left\{u_{1}, u_{4}, u_{3}\right\}$ is a secure dominating set. Therefore, $\gamma_{s r}(G)=5<\gamma_{s}(G)+\operatorname{dim}(G)=6$ and $\max \left\{\gamma_{s}(G), \operatorname{dim}(G), \gamma_{r}(G)\right\}=4<\gamma_{s r}(G)=5$.

Remark 6. When $G=K_{n}, \gamma(G)=1, \gamma_{s}(G)=1, \operatorname{dim}(G)=n-1, \gamma_{r}(G)=n-1, \gamma_{s r}(G)=n-1$. Therefore, $\max \left\{\gamma_{s}(G), \operatorname{dim}(G), \gamma_{r}(G)\right\}=\gamma_{s r}(G)$.

Observation 3. $\gamma_{s r}(G) \geq g(m, d)$, where $g(m, d)=\min \left\{t: t+\sum_{i=1}^{t}\left(\begin{array}{l}t \\ i\end{array}\right)(d-1)^{t-i} \geq m\right\}$, d is a diameter of $G$, the order of $G$ is $m \geq 2$, and $d$ and $m$ are positive integers with $d<m$. This follows from proposition 2.1 [6] and that $\gamma_{s r}(G) \geq \gamma_{r}(G)$.

Observation 4. For every positive integer $k$, there are only finitely many connected graphs with secure resolving domination number $k$.

Proof. Consider a graph $G$ with order $m \geq 2$ and $\gamma_{s r}(G)=k$. From corollary 2.2 [6] $m \leq k+$ $\sum_{i=1}^{k}\left(\begin{array}{c}k \\ i\end{array}\right)(d-1)^{l-i} \cdot \gamma(G) \leq \gamma_{s r}(G)=k$. Therefore, the diameter of $G$ is not more than $3 k-1$. Therefore, $m \leq k+\sum_{i=1}^{k}\left(\begin{array}{c}k \\ i\end{array}\right)(3 k-2)^{k-i}$. Therefore, there are only finitely many connected graphs with $\gamma_{s r}(G)=k$

Remark 7. Suppose that $\gamma_{s r}(G)=2$. Then, the number of connected graphs with $\gamma_{s r}(G)=2$ has an order of at most 11 .

Proof. By the above observation, $n \leq 2+\sum_{i=1}^{2}\left(\begin{array}{c}2 \\ i\end{array}\right)(6-2)^{2-i}=2+\left(\begin{array}{c}2 \\ 1\end{array}\right) 4+\left(\begin{array}{c}2 \\ 2\end{array}\right) 4=2+8+$ $1=11$.

In fact, the above bound for $n$ can be improved.

Observation 5. For any $G$ with $\gamma_{s r}(G)=2$, the order of $G$ is not more than 4. 
Proof. Let $\gamma_{s r}(G)=2$. Let $X=\{p, q\}$ be a $\gamma_{s r}$-set of $G$. If $d(p, q) \geq 4$, then $p$ and $q$ cannot dominate the point at a distance 2 from $p$ in the shortest path joining $p$ and $q$. Therefore, $d(p, q) \leq 3$.

Case (i): Distance between $p$ and $q$ is 1 .

As every single vertex in $V(G)-X$ is adjacent with either both $p$ and $q$ or one of them, the distances of the vertices in $V(G)-X$ from $p$ and $q$ are $(1,2),(2,1)$, and $(1,1)$. Then, $G$ is as follows:

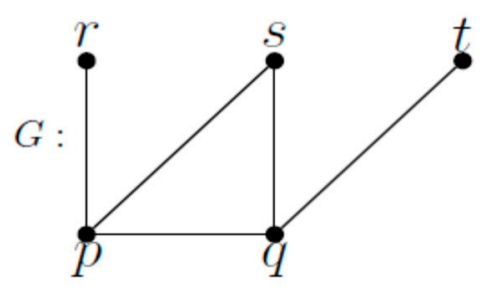

Here, $s$ cannot enter $X$ by removing a vertex of $X$, since such a resulting set is not a dominating set. Therefore, $G=P_{4}$. If both pendants $r$ and $t$ are removed, then the resulting set is $K_{3}$, for which $\gamma_{s r}(G)=2$. That is, $G=K_{3}$.

If $r, s$, and $t$ are present and $r$ is adjacent with $s$, then the graph is:

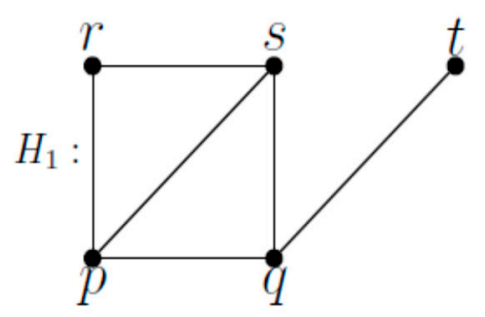

Here, $r$ cannot enter $X$, since resolving fails.

If $r, s$, and $t$ are existing, $r$ and $t$ are adjacent with $s$, then the graph is:

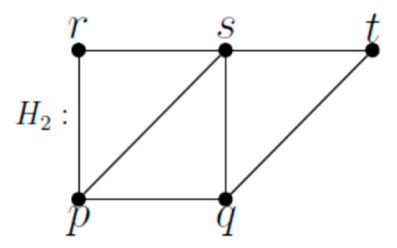

Here, $s$ cannot enter $X$, since resolution fails.

If $r$ and $t$ are adjacent, then the graph $H_{3}$ is as follows:

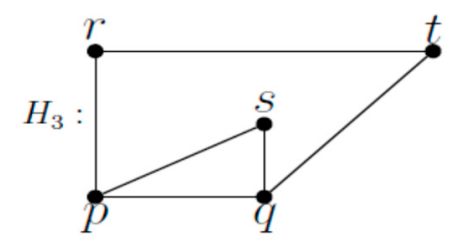

In $H_{3}, s$ cannot enter $X$, since domination fails. If $r, s$, and $t$ are mutually adjacent, then the graph $\mathrm{H}_{4}$ is as follows:

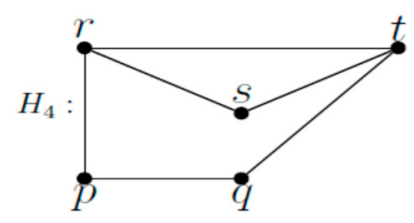


In the above graph, $s$ cannot enter $X$, since resolution fails. The remaining cases are: (i) $s$ is not present, and $r$ and $t$ are non-adjacent. In this case, $G=P_{4}$. (ii) $r$ and $t$ are available, $s$ is not present and $r$ and $t$ are adjacent. We get $G=C_{4}$. (iii) $r$ and $s$ are alone present and $r$ and $s$ adjacent. We get $C_{4}$ with a diagonal. (iv) $r$ and $s$ are alone and present, and they are not adjacent. We get $K_{3}$ with a pendant vertex. Thus, in this case, $G=P_{3}, P_{4}, C_{4}, C_{4}$ with a diagonal and $K_{3}$ with a pendant vertex.

Case (ii): $d(p, q)=2$.

Since every vertex in $V(G)-X$ is adjacent with at least one of $p$ and $q$, the distances of the vertices 3 in $V(G)-X$ from $p$ and $q$ are $(1,3),(3,1),(1,2),(2,1)$, and $(1,1)$. Therefore, the graph is as follows:

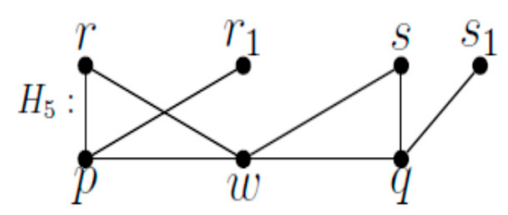

For security in $H_{5}, r_{1}$ cannot enter $\{p, q\}$ by removing $p$ or $q$, since domination fails. Therefore, only one of $r$ and $r_{1}$ can be present. Similarly, one of $s$ and $s_{1}$ can be present. Therefore, the graphs are as follows:

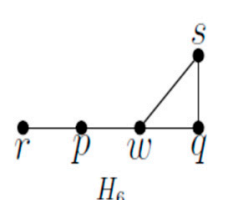

$H_{6}$

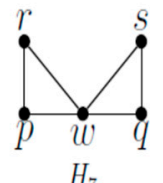

$\mathrm{H}_{7}$
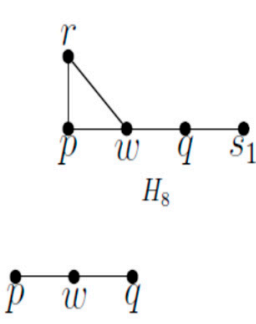

$H_{10}$
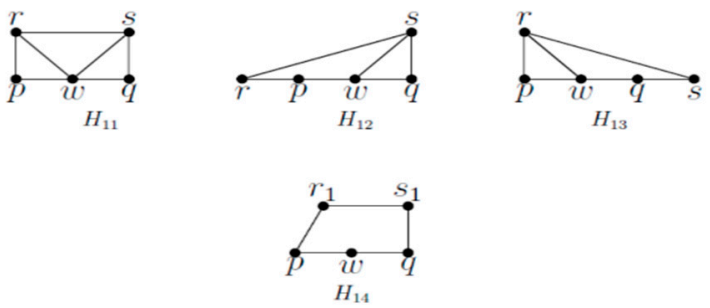

In graph $H_{9}, w$ cannot enter $X=\{p, q\}$. In $H_{6}$, if $w$ enters $X$ by removing $p$ or $q$, then the resulting set is not resolving, although it is dominating. In graph $H_{7}$, if $w$ enters $X$, then for domination, $q$ should be replaced by $w$. However, the resulting set is not resolving. Same is the graph $\mathrm{H}_{8}$. In graphs $H_{11}, H_{12}$, and $H_{13}, w$ cannot enter $X$, since resolution fails. In graph $H_{14}, w$ cannot enter $X$, since domination fails.

Case (iii): $d(p, q)=3$.

Since vertices in $V(G)-X$ are adjacent with one of $p$ and $q$, the distances of the vertices in $V(G)-X$ from $p$ and $q$ are $(1,1),(1,2),(2,1),(1,3),(3,1),(1,4)$, and $(4,1)$.

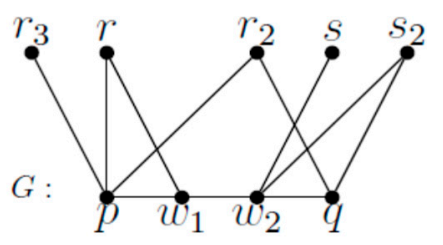

Only one of $r, r_{2}$, and $r_{3}$ can be present, since $\{p, q\}$ is an SRD set. Similarly, only one of $s$ and $s_{2}$ can be present. If any number of edges among the vertices $r_{3}, r, x_{2}, s$, and $s_{2}$ are inserted, then $w_{1}$ cannot enter $X$ by replacing $p$ or $q$, since domination fails. 
Subcase (i): $r_{3}$ is present.

In this case, $w_{1}$ cannot enter $X$ by replacing $p, q$, since domination fails.

Subcase (ii): $s_{2}$ is exist.

In this case, $w_{2}$ cannot enter $X$ by replacing $p, q$. (since domination fails).

Subcase (iii): One of $r, r_{2}$, and $\mathrm{s}$ is present.

Then, the graphs are as follows:
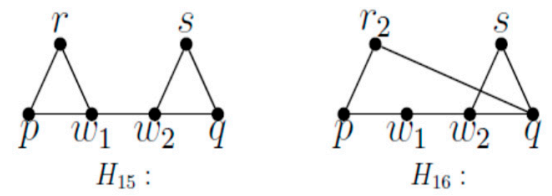

In $\mathrm{H}_{15}$, either $w_{1}$ or $w_{2}$ cannot enter $X$ by replacing $p, q$, since resolution fails. In $H_{16}$, $w_{1}$ cannot enter $X$, since domination fails.

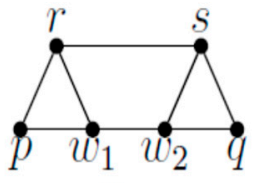

$$
H_{17} \text { : }
$$

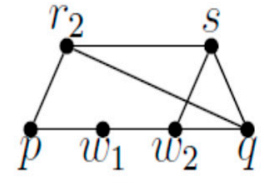

$H_{18}$ :

In $\mathrm{H}_{17}, w_{1}$ cannot enter $X$, since resolution fails. In $H_{18}, w_{1}$ cannot enter $X$, since domination fails.

Subcase (iv): Only one of $r, r_{2}$ is present, and none of $s, s_{2}$ is present.

Then, the graphs are as follows:
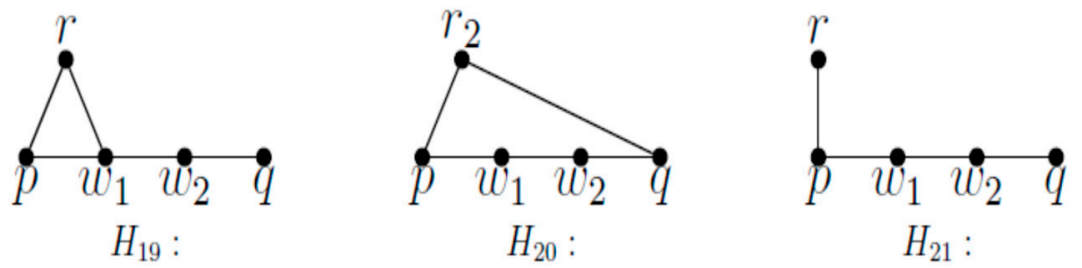

In $H_{19}, w_{1}$ cannot enter $X$, since resolution fails. In $H_{20}, w_{2}$ cannot enter $X$, since domination fails. In $H_{21}, w_{1}$ cannot enter $X$, since domination fails. Similarly, if only one of $s$ and $s_{2}$ is present, and none of $r, r_{1}$, and $r_{2}$ is present, then $w_{2}$ cannot enter $X$. Therefore, $G=P_{4}$.

Subcase (v): None of $x, x_{2}, x_{3}, y$, and $y_{2}$ is present. Then, $G=P_{4}$.

Corollary 2. $\gamma_{s r}(G)=2$ if and only if $G=P_{4}, P_{3}, C_{3}, C_{4}$, and $K_{3}$ with a pendant vertex and $K_{4}-\{e\}$.

Proposition 2. Let $l \geq 1, m \geq 2$, and $n=l+m$ be three integers. Then, there exists $G$ with $\gamma(G)=l$, $\operatorname{dim}(G)=q$ and $\gamma_{s r}(G)=n$.

Proof. We follow the proof given in proposition 3.1 [6]. Construct a graph $G$ from the path $P_{3 l-1}$ : $v_{1}, v_{2}, \ldots, v_{3 l-1}$ of order $3 l-1$. Join $m$-pairs of vertices $x_{j}, y_{j}, 1 \leq j \leq m$ and join $x_{j}$ and $y_{j}$ for each $j$. Consider, $F_{j}$ - a copy of the path $P_{2}: x_{j} y_{j}$. Join the vertex of $F_{j}, 1 \leq j \leq m$ to the vertex $v_{3 t-1}$. For $l=m=2$, the graph is as follows:

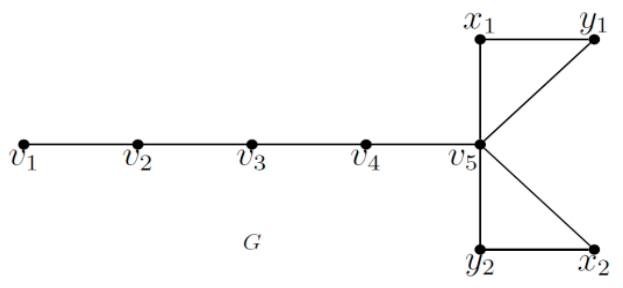


Let $\mathrm{V}=\left\{v_{1}, v_{2}, \ldots, v_{3 l-1}\right\}, T=\left\{x_{1}, x_{2}, \ldots, x_{m}\right\}$, and $W=\left\{y_{1}, y_{2}, \ldots, y_{m}\right\}$. Then, $\gamma(G)=l$ and $\operatorname{dim}(G)=m$ (since $\left\{v_{2}, v_{5}, \ldots, v_{3 l-1}\right\}$ is dominating, and $T$ is a basis of $G$. Each resolving set of $G$ has at least one vertex from each set, $\left\{x_{j}, y_{j}\right\}, 1 \leq j \leq m$. All of the vertices $x_{j}, y_{j}$, and $v_{3 l-1}$ are dominated by them. We need at least $\frac{3 l-2}{3}=l$ vertices to dominate $V-\left\{v_{3 l-1}\right\}$. As a result, $\gamma_{r}(G) \geq l+m$. However, $K=\left\{v_{2}, v_{5}, \ldots, v_{3 l-1}\right\} \cup X$ is a resolving dominating set for $G$. Hence, $\gamma_{r}(G) \leq|K|=l+m$. Therefore, $\gamma_{r}(G)=l+m$. Clearly, $K$ is a SRD set of $G$. Therefore, $\gamma_{s r}(G) \leq l+m$. However, $\gamma_{s r}(G) \geq \gamma_{r}(G)=l+m$. Therefore, $\gamma_{s r}(G)=l+m=n$.

Theorem 4. Let $G$ be a graph of order $n \geq 2$. $\gamma_{s r}(G)=n-1$ if and only if $G=K_{n}$ or $K_{1, n-1}$.

Proof. $\gamma_{s r}(G)=n-1$. Consequently, no $(n-2)$ subset of $V(G)$ is a SRD set of $G$. Suppose that there exists an $(n-2)$ resolving subset $S$ of $V(G)$ that is not a secure dominating set of G. Let $V(G)-S=$ $\{u, v\}$. Suppose that $S$ is not a dominating set of $G$. Since $G$ is connected, exactly one of $u$ and $v$ is not dominated by $S$, say $u$. Clearly, $u$ is a pendant of $v$.

Claim: $v$ is adjacent with every vertex of $S$.

Suppose that $v$ is not adjacent with a vertex $w$ of $S$. Let $T^{\prime}=(S-\{w\}) \cup\{v\}=V(G)-\{u, w\}$. Since $G$ is connected and $w$ is not adjacent with $u$ and $v, w$ is adjacent with some vertex of $S$. $T^{\prime}$ is a dominating set of $G$. Therefore, there exists an $(n-2)$ subset that is a resolving and dominating set of $G$.

$S_{1}=\left(T^{\prime} \cup\{u\}\right)-\{v\}$ is a dominating set of $G$. Clearly, $S$ is a resolving set, since $d(u, v)=1, d(u$, $w) \geq 2$. Therefore, $S_{1}$ is a secure resolving domination set of $G$. Therefore, $\gamma_{s r}(G) \leq n-2$, which is a contradiction. $w$ is adjacent with some vertex $x$ in $S$. Therefore, $S_{2}=(S \cup\{w\})-\{x\}$ is a dominating set of $G . d(u, v) \geq 2$ and $d(x, w)=1$. Therefore, $S_{2}$ is a secure resolving domination set of $G$. Therefore, $\gamma_{s r}(G) \leq n-2$, which is a contradiction. Suppose that $S$ is a dominating set of $G$, but not a secure dominating set of $G$. Suppose that $u$ cannot enter $S$ by replacing a vertex of $S$. Then, any neighbor of $u$ is either an isolate of $S$ or has private neighbor $v$. Suppose that every neighbor $x$ of $u$ is an isolate of $S$. In this case, if $u$ is not adjacent with $v$, then $G$ is disconnected, which is a contradiction. If $u$ is adjacent with $v$, then $(S-N[u]) \cup\{v\}$ is connected. Then, $(S-\{x\}) \cup\{u\}$ is a dominating set of $G$.

Suppose that $(S-N(u))=\phi$. Then, either $G$ is a star or $G$ is of the form:

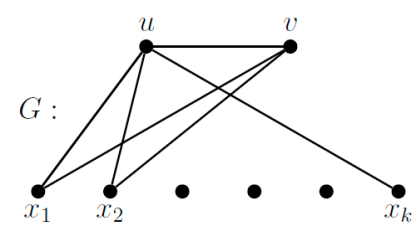

where $v$ is adjacent with some or all of $x_{1}, x_{2}, \ldots, x_{k}$. If $G$ is a star, then $\gamma_{s r}(G)=n-1$. If $G$ is not a star, then the above graph has $\gamma_{s r}(G) \leq n-2$, which is a contradiction.

Suppose that $(S-N(u)) \neq \phi$. Then, $v$ is adjacent with at least one vertex, say $z$ of $(S-N(u))$. $d(v, z)$ $=1, d\left(x_{i}, z\right) \neq 1$. Therefore, $\left(S-\left\{x_{i}\right\}\right) \cup\{u\}$ is resolving. Therefore, there exists an $(n-2)$ SRD set of $G$, which is a contradiction.

Suppose that there exists a neighbor $x$ of $u$ which has private neighbor $v$. Let $x$ be an isolate of $S$. Then, $G$ is of the form $H_{1}$ or of the form $H_{2}$, where $u$ and $v$ are made adjacent in $H_{1}$. However, $H_{1}$ and $H_{2}$ have an $(n-2)$ secure dominating set of $G$, which is a contradiction.

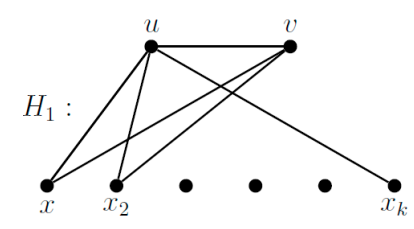


If $x$ is not an isolate of $S$, then either $G$ is complete, or $G$ has $(n-2)$ SRD set of $G$, which is a contradiction. Similarly, $v$ can enter $S$ by replacing a vertex of $S$. Therefore, any $(n-2)$ resolving subset of $V(G)$ is a secure dominating set of $G$, provided that $G$ is not a star or $G$ is not $K_{n}$.

Therefore, the theorem follows.

\section{Discussion and Conclusions}

A study of SR sets and SRD sets is initiated in this paper. Further work may be done on (i) conditions for the minimality of SR sets (SRD sets), (ii) uniform SR set (SRD set) (that is to find the least positive integer $t$ such that every subset of $V(G)$ of cardinality $t$ is an SR set (SRD set)), (iii) a study of secure metric resolving sets (metric resolving dominating sets) in a graph, and (iv) secure independent resolving sets (secure independent resolving dominating sets).

Author Contributions: H.S. first author is responsible for the preparation of an article, review, editing and visualization under the guidance of S.A.

Funding: This research is carried out without any external financial support.

Conflicts of Interest: The authors confirm no conflict of interest.

\section{References}

1. Chartrand, G.; Eroh, L.; Johnson, M.A.; Oellermann, O.R. Resolvability in graphs and the metric dimension of a graph. Discret. Appl. Math. 2000, 105, 99-113. [CrossRef]

2. Slater, P.J. Leaves of trees. In Proceedings of the 6th Southeast Conference on Combinatorics, Graph Theory and Computing, Boca Raton, FL, USA, 17-20 February 1975; pp. 549-559.

3. Slater, P.J. Dominating and reference sets in graphs. J. Math. Phys. 1988, 22, 445-455.

4. Brigham, R.C.; Chartrand, G.; Dutton, R.D.; Zhang, P. Resolving Domination in Graphs. Math. Bohem. 2003, $128,25-36$.

5. Harary, F.; Melter, R.A. On the metric dimension of graph. Ars Comb. 1976, 24, 191-195.

6. Cockayne, E.J.; Favaron, O.; Mynhardt, C.M. Secure domination, weak roman domination and forbidden subgraph. Bull. Inst. Comb. Appl. 2003, 39, 87-100.

(C) 2018 by the authors. Licensee MDPI, Basel, Switzerland. This article is an open access article distributed under the terms and conditions of the Creative Commons Attribution (CC BY) license (http://creativecommons.org/licenses/by/4.0/). 